\title{
Impresión diagnóstica clínico-radiológica de pituicitoma, presentación de dos casos clínicos
}

\author{
Paula Andrea Moreno Lucero'; Angélica María Fonseca Niño²; William Rojas García ${ }^{3}$; María \\ Claudia Rivera ${ }^{4}$
}

${ }^{1}$ Estudiante de XII semestre de la Fundación Universitaria Ciencias de la Salud del Hospital de San José. Bogotá D.C., Colombia. ${ }^{2}$ Estudiante de XI semestre de la Fundación Universitaria Ciencias de la Salud del Hospital de San José. Bogotá D.C., Colombia.

${ }^{3}$ Médico internista, Endocrinólogo y profesor asociado de la Fundación Universitaria Ciencias de la Salud del Hospital de San José. Bogotá D.C., Colombia.

${ }^{4}$ Médico internista, fellow endocrinología de la Fundación Universitaria Ciencias de la Salud del Hospital de San José. Bogotá D.C., Colombia.

Declaración de conflicto de interés

Ninguno de los autores declara conflicto de interés.

Fecha de recepción: 4/04/2016

Fecha de aceptación: 6/07/2016

\section{Resumen}

$\mathrm{P}$ ituicitoma es un tumor único de bajo grado, originado en las células magnocelulares de la neurohipófisis, o en el infundíbulo. Es una entidad rara, que afecta a los adultos. Las manifestaciones clínicas dependen de la localización y el tamaño, pudiendo simular un adenoma hipofisiario, pues también puede comprometer cualquier eje hipofisiario. El diagnóstico se realiza por medio de resonancia magnética nuclear (RMN) y biopsia, y su tratamiento puede ser por medio de medicamentos o cirugía.

En este articulo presentamos dos casos clínicos de dos adultos con pituicitoma, quienes tuvieron hallazgos radiológicos similares.

Palabras claves: pituicitoma, hipófisis posterior, neoplasias pituitarias, adulto joven.

\section{Abstract}

Pituicytoma is an only low-grade tumor which is originated from magnocellular cells in neurohypophysis or in infundibulum. It is a rare condition that affects adults. The clinical manifestations depends on the location and size because it is similar to a pituitary adenoma, so it produces nonspecific damage to the pituitary axis. For the diagnosis is usually performed with Magnetic Resonance Imaging (MRI) and biopsy and treatment of this disease may be with drugs or surgery.
In this article we show two clinical cases of two adults with pituicytoma who had similar radiological findings.

Key words: pituicytoma, posterior pituitary, pituitary tumors, adult.

\section{Introducción}

La hipófisis o glándula pituitaria reside en la silla turca del hueso esfenoides en la base del cráneo; está dividida en dos zonas, la adenohipofisis (secreta ACTH, GH, PRL, TSH, LH y FSH) y la neurohipófisis (compuesta por la pars nervosa e infundíbulo almacena hormonas antidiurética y oxitocina); zonas fácilmente distinguidas en una resonancia nuclear magnética (RMN) cerebral. Adicionalmente, la glándula está regulada por liberación de factores u hormonas hipofisiotrópicas, efectos de retroalimentación de hormonas circulantes y secreciones para y autocrinas ${ }^{(1,2)}$, por lo anterior si hay alguna alteración sobre la estructura de la glándula se puede producir disfunción de la misma, como puede ser el caso de la patología tumoral.

El pituicitoma es una neoplasia selar rara, sólida, con contornos definidos y de bajo grado (clasificación de la organización mundial de la salud (OMS) grado I), proveniente del tejido glial o de sostén (pituicitos) ubicado en las zonas perivasculares de la neurohipófisis ${ }^{(3-5)}$.

Resulta difícil determinar la prevalencia de esta patología debido a que antes del 2007, con el término pituicitoma, fueron nombrados diversas clases de tumores localizados en la región selar ${ }^{(3)}$; sin embargo, con los casos de pituicitoma reportados actualmente se ha establecido una relación hombremujer de 1,6:1 ${ }^{(6,7)}$ siendo más frecuente en hombres adultos ${ }^{(6)}$. El grupo de la Sociedad Alemana de Endocrinología solamente hizo diagnóstico de pituicitoma a 5 casos con respecto a 6.739 pacientes con lesiones en región selar desde 1996 hasta $2009^{(8)}$. No hay datos oficiales en Colombia de casos reportados hasta el momento.

A continuación, presentaremos los casos de dos adultos jóvenes en quienes se sugiere esta entidad como principal sospecha diagnóstica. 


\section{Presentación de casos}

\section{Primer caso}

Al servicio de endocrinología, ingresa paciente de género masculino de 38 años, procedente de Puerto López (Meta, Colombia) con antecedente de infertilidad, disminución de la libido, y de las eyaculaciones, asociado a disfunción sexual. Al examen físico se evidenció hipogonadismo, signos de resistencia a la insulina y síntomas de hipotiroidismo (aumento de peso, astenia, adinamia, sensibilidad al frío). El paciente trae reporte de espermograma con hallazgos de azoospermia e hipospermia, paraclínicos que evidencian supresión de todos los ejes hipofisiarios sin hiperprolactinemia (tabla 1) y RMN de silla turca donde llama la atención ausencia de neurohipófisis y diagnóstico presuntivo de pituicitoma (figura 1).

Se hace diagnóstico de hipopituitarismo secundario a pituicitoma, y se realizó test de estimulación de LH con administración de $\mathrm{GnRH}$, confirmando compromiso de tallo hipofisiario y no de células gonadotropas hipofisiarias por elevación de LH post estímulo (tabla 2).

Por lo anterior, se inició manejo con levotiroxina $100 \mathrm{mcg}$ al día, metformina $850 \mathrm{mg}$ cada 8 horas con cada comida y enantato de testosterona $250 \mathrm{mg}$ mensual. Al año de trata-
Figura 1. RMN de silla turca contrastada

Contorno superior cóncavo, con altura máxima de $4 \mathrm{~mm}$, no se identifica neurohipófisis. Imagen de masa dependiente del tallo hipofisiario en su aspecto posterosuperior, homogénea. Presenta isointensidad en T1 y T2 e hipercaptación con el medio de contraste. Tiene dimensiones máximas aproximadas: longitud: 4,7 $\mathrm{mm} x$ antero posterior: $7 \mathrm{~mm} \times$ altura: $4 \mathrm{~mm}$ (ver flecha).

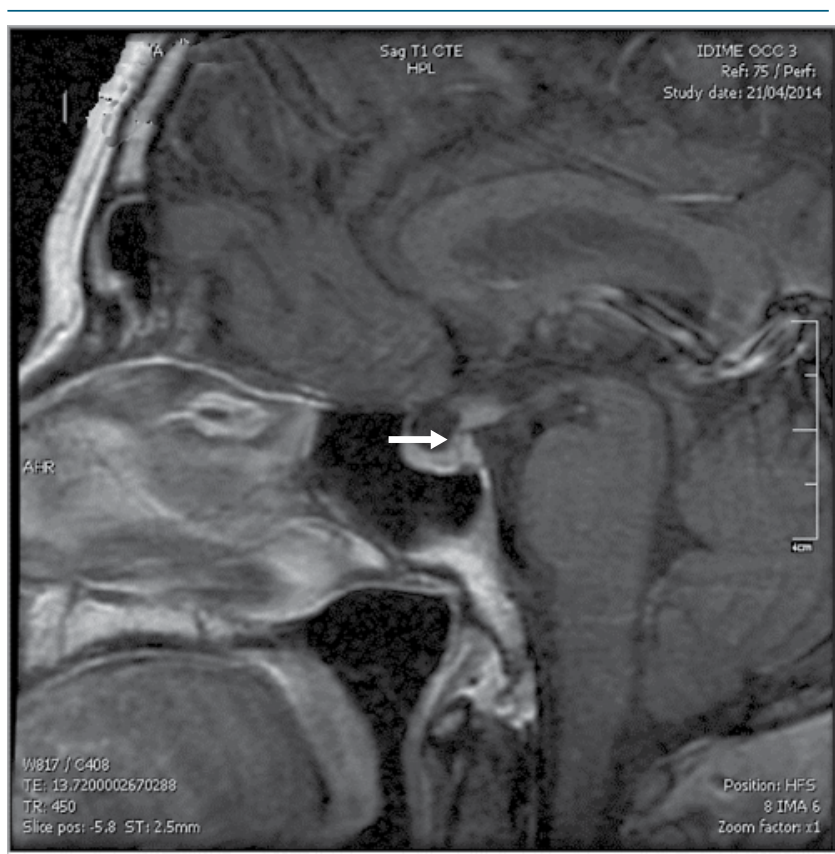

Tabla 1. Paraclínicos de inicio y control del primer caso

\begin{tabular}{|c|c|c|c|}
\hline & \multicolumn{2}{|c|}{ Valores } & \multirow{2}{*}{$\begin{array}{l}\text { Rangos referencia } \\
\text { para varón }\end{array}$} \\
\hline & 2014 & 2015 & \\
\hline $\mathrm{FSH}(\mathrm{mUI} / \mathrm{mL})$ & 0,45 & & $1,5-12,4$ \\
\hline $\mathrm{LH}(\mathrm{mUl} / \mathrm{mL})$ & 0,36 & & $1,7-8,6$ \\
\hline Testosterona libre (pg/mL) & 1,26 & 6,905 & $312-1.041$ \\
\hline Testosterona total (ng/mL) & 0,42 & 0,72 & $2,8-8$ \\
\hline $\begin{array}{l}\text { Proteína transportadora de } \\
\text { hormonas sexuales ( } \mathrm{nmol} / \mathrm{L} \text { ) }\end{array}$ & 23,7 & & $15-100$ \\
\hline ACTH (pmol/L) & 35 & & $1,3-16,7$ \\
\hline Cortisol 8 am (g/dL) & 15,97 & & $5-25$ \\
\hline $\begin{array}{l}\text { Cortisol en orina de } 24 \text { horas } \\
\text { (nmol/24 h) }\end{array}$ & 367 & 218,72 & $50-190$ \\
\hline Prolactina (ng/mL) & 14,4 & & \\
\hline $\begin{array}{l}\text { Espermograma (recuento de } \\
\text { espermatozoides) }\end{array}$ & 0 & & \\
\hline Glicemia basal (mg/dL) & 139,7 & 105,6 & $<126$ \\
\hline $\mathrm{GH}(\mathrm{ng} / \mathrm{mL})$ & 0,05 & & $1-9$ \\
\hline Potasio (Meq/mL) & 4,8 & & $3,5-4,5$ \\
\hline Sodio (Meq/mL) & 147 & & $135-145$ \\
\hline Hemoglobina glicosilada (\%) & 6,2 & 5,9 & $<6,5 \%$ \\
\hline TSH ( mUl/L) & 0,005 & 2,27 & $0,34-4,25$ \\
\hline T4L ( ng/dL) & & 0,83 & $0,7-1,24$ \\
\hline
\end{tabular}

miento el paciente refiere mejoría de la libido, sin embargo, con deseo de fertilidad y debido a riesgo de atrofia testicular se cambió el enantato de testosterona a gonadotropina coriónica con dosis de 1000 unidades interdiarias. En el momento, sin lograr concepción.

Actualmente, el paciente continúa en control semestral con el endocrinólogo para manejo de la alteración de ejes hipofisiarios, control metabólico y así posteriormente ser llevado a resección quirúrgica por parte del servicio de neurocirugía.

\section{Segundo caso}

Al servicio de Endocrinología del Hospital de San José ingresa una paciente de género femenino de 26 años que consulta por polidipsia, poliuria, deshidratación mucocutánea, aumento de peso y cefalea persistente asociado a náuseas desde hace dos años, además refiere amenorrea sin galactorrea, sin respuesta a manejo médico planteado por ginecología. Con antecedente de hipotiroidismo en suplencia hormonal con levotiroxina $50 \mathrm{mcg} /$ día. Al examen físico se evidenció mucosa oral seca y obesidad grado I (IMC 31,8). Consigo trajo reporte de RMN cerebral extrainstitucional que evidenciaba ausencia de neurohipófisis con presencia de 
Tabla 2. Test de estimulación con GnRH primer caso

LH pre y post prueba de estimulación con GnRH
\begin{tabular}{l|c} 
LH basal (mUl/mL) & 0,24 \\
\hline LH a los 30 minutos (mUl/mL) & 6,45 \\
\hline LH a los 60 minutos $(\mathrm{mUl} / \mathrm{mL})$ & 8,03 \\
\hline LH a los 90 minutos $(\mathrm{mUl} / \mathrm{mL})$ & 7,48 \\
\hline
\end{tabular}

Figura 2. RMN de silla turca contrastada

Masa en región hipotalámica retroquiasmatica aparentemente relacionada con el infundíbulo con diámetro máximo de $10 \mathrm{~mm}$, ausencia de neurohipofisis se sugiere pituicitoma como primera posibilidad (ver flecha).

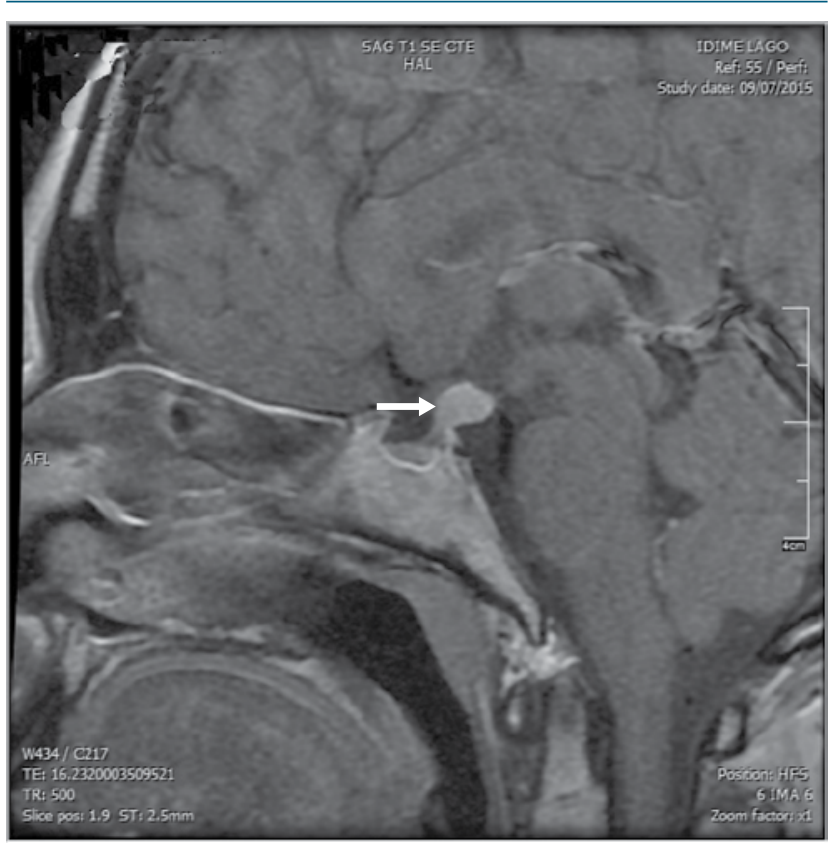

pituicitoma (figura 2), además paraclínicos con hallazgos de densidad urinaria disminuida (1.003), hipernatremia (148 $\mathrm{mEq} / \mathrm{ml})$, hipercalemia $(5,3 \mathrm{mEq} / \mathrm{ml})$, hiperprolactinemia (143,8 ng/ml), TSH y T4 libre normales (3,36 mUI/L y 0,8 ng/ dl), (respectivamente con suplemento hormonal), estradiol disminuido (5 ng/L), cortisol a.m. en rangos normales $(11,1$ $\mathrm{mcg} / \mathrm{dL})$, somatomedina C normal $(82,3 \mu \mathrm{g} / \mathrm{L})$, FSH y LH disminuida $(2,46 \mathrm{mUI} / \mathrm{mL}$ y $0,49 \mathrm{mUI} / \mathrm{mL}$, respectivamente) y glucemia normal (87 mg/dL); por lo anterior se concluye que la paciente presenta hipogonadismo hipogonadotrófico con hipotiroidismo central y sospecha de diabetes insípida de origen central, secundarios a pituicitoma. Se consideró que hiperprolactinemia se debía a ubicación del tumor.

Debido a lo anterior se inició manejo con estrógenos conjugados más progesterona. Se realizó test de restricción hidrica (tabla 3) que confirma sospecha de diabetes insípida.

Se adicionó al manejo médico desmopresina vía oral 120 mcg cada 12 horas. En controles posteriores, la paciente ha re-
Tabla 3. Paraclínicos de inicio y control del segundo caso

\begin{tabular}{l|c|c}
\multicolumn{1}{c|}{ Paraclínicos } & $\begin{array}{c}\text { Pretest de } \\
\text { restricción } \\
\text { hídrica }\end{array}$ & $\begin{array}{c}30 \text { minutos } \\
\text { postest de } \\
\text { restricción } \\
\text { hídrica }\end{array}$ \\
\hline Glicemia (mg/dL) & 92 & 87 \\
\hline $\begin{array}{l}\text { Potasio sérico } \\
\text { (mmol/L) }\end{array}$ & 4,8 & 4,5 \\
\hline Creatinina (mg/dL) & 0,8 & 0,7 \\
\hline BUN (mg/dL) & 9 & 9 \\
\hline $\begin{array}{l}\text { Sodio sérico } \\
\text { (mmol/L) }\end{array}$ & 153 & 152 \\
\hline $\begin{array}{l}\text { Osmolaridad } \\
\text { plasmática } \\
\text { (mOsm/L) }\end{array}$ & 292,9 & \\
\hline $\begin{array}{l}\text { Glucosa en orina } \\
\text { (mg/dl) }\end{array}$ & 20 & \\
\hline $\begin{array}{l}\text { Densidad urinaria } \\
\text { (tira reactiva) }\end{array}$ & 1005 & \\
\hline $\begin{array}{l}\text { Osmolaridad } \\
\text { urinaria (mOsm/L) }\end{array}$ & 83,8 & \\
\hline $\begin{array}{l}\text { Sodio urinario } \\
\text { mmol/L }\end{array}$ & 31 & \\
\hline
\end{tabular}

ferido mejoría de sintomatología con estabilización de sodio sérico y densidad urinaria.

Se continúa en seguimiento trimestral para control de los ejes tirotropo, somatotropo y gonadotropo.

\section{Discusión}

Pituicitoma es un tumor benigno, poco frecuente, originado en la neurohipófisis o el infundíbulo, se caracteriza por ser una masa sólida, bien circunscrita localizada en la región selar, supraselar o ambas. Esta entidad fue descrita por primera vez, por Liss en $1958^{(6,9)}$. Sin embargo, el término fue utilizado para nombrar diversos tumores en la misma localización, incluyendo los tumores granulares y astrocitomas pilocíticos ${ }^{(6)}$. En el 2007, la OMS clasificó al pituicitoma como patología independiente a estas últimas ${ }^{(4)}$.

Se han formulado dos teorías con el propósito de explicar su origen, la primera de ellas describe que el pituicitoma proviene de los pituicitos, los cuales son células de la microglia modificadas que se encuentran en áreas perivasculares y participan en la liberación de hormonas hipotalámicas.

Una teoría alternativa sugiere que los pituicitomas derivan de células fusocelulares de la adenohipófisis, comportándose como células madre, dando lugar a células diferenciadas con comportamiento endocrino ${ }^{(6)}$.

Las manifestaciones clínicas son secundarias al efecto de masa, producido por el aumento de tamaño del tumor, siendo 
las más frecuentes cefalea en 38,9\% de los casos, disturbios visuales en $31,5 \%$ de los casos, y alteración en la liberación de hormonas hipofisiarias en 31,5\% de los casos, y de esta última, los hallazgos más frecuentes son disminución de la libido e hipogonadismo hipogonadotrófico, y menos frecuente, la presencia de diabetes insípida $(1 \%)^{(3,7,10,13,14)}$. En nuestros casos ambos pacientes cursaron con hipogonadismo hipogonadotrófico asociado a hipotiroidismo central, el caso 2 cursa además con hiperprolactinemia, siendo de gran particularidad, puesto que es menos frecuente la presencia de diabetes insípida.

A pesar de que no existen hallazgos radiológicos patognomónicos de esta lesión, la tomografía axial computarizada (TAC) y la resonancia magnética nuclear (RMN) ayudan a hacer una aproximación diagnóstica. En la TAC se puede encontrar una lesión sólida bien circunscrita, mientras que en la RMN se puede evidenciar una lesión isointensa en T1 e hiperintensa en $\mathrm{T} 2^{(6,11)}$, estos últimos compatibles con los hallazgos en nuestro caso número 1.

Sin embargo, otros tumores de la región selar y supraselar deben ser considerados como diagnósticos diferenciales, por ejemplo, meningiomas, craneofaringiomas, hemangiopericitomas, tumores de células germinales, hamartomas, gangliomas, sarcoidosis, astrocitomas, y tumores metastásicos ${ }^{(6)}$. A pesar de que el pituicitoma presenta alto riesgo de hemorragia, si en imágenes se evidencia esta en región selar, se sugiere incluir otros posibles diagnósticos diferenciales, como son los quistes de la bolsa de Rathke, el angioma cavernoso, el angioma venoso o el aneurisma venoso ${ }^{(6,12)}$.

El tratamiento de elección es resección del tumor, no obstante, debido al riesgo de sangrado inesperado o a adherencia a estructuras adyacentes, se prefiere realizar resección subtotal, a pesar del riesgo de reproducción de la lesión ${ }^{(6)}$.

A nivel histológico, los pituicitomas se caracterizan por tener células en forma de huso, núcleo oval, citoplasma eosinófilo y homogéneo, poca o ninguna granulación. Los marcadores de inmunohistoquímica con los que con mayor frecuencia se encuentra inmunorreactividad son proteína S-100 y vimentina ${ }^{(6)}$.

Nos encontramos a la espera de la resección quirúrgica de nuestros casos, para confirmación patológica e inmunohistoquímica.

\section{Referencias}

1. Low MJ. Neuroendocrinology. In: Elsevier I, editor. Williams Textbook of Endocrinology. 13: Elsevier, Inc.; 2016. p. 109-75.

2. R. B, Javorsky M, MD DCA, MS J, W. Findling M, J. Blake Tyrrell M. Hipotálamo y glándula hipófisis GreenspanEndocrinologiaBasicayClinica. 9. 9 ed. México, D.F.: McGRAW-HILL/Interamericana Editores, S.A. de C.V; 2011. p. 65-114.

3. Rivero-Celada D, Barrera-Rojas M, Orduna-Martínez J, Lorente-Muñoz A, Alfaro-Torres J, Alberdi-Viñas J. [Pituitary pituicytoma]. Neurocirugia (Astur). 2012;23(4):165-9.

4. Louis DN, Ohgaki H, Wiestler OD, Cavenee WK, Burger PC, Jouvet A, et al. The 2007 WHO classification of tumours of the central nervous system. Acta Neuropathol. 2007;114(2):97-109.

5. Kosuge Y, Hiramoto J, Morishima H, Tanaka Y, Hashimoto T. Neuroimaging characteristics and growth pattern on magnetic resonance imaging in a 52-year-old man presenting with pituicytoma: a case report. J Med Case Rep. 2012;6:306.

6. Secci F, Merciadri P, Rossi DC, D’Andrea A, Zona G. Pituicytomas: radiological findings, clinical behavior and surgical management. Acta Neurochir (Wien). 2012;154(4):649-57; discussion 57.
7. Chu J, Yang Z, Meng Q, Yang J. Pituicytoma: case report and literature review. Br J Radiol. 2011;84(999):e55-7.

8. Pirayesh Islamian A, Buslei R, Saeger W, Fahlbusch R. Pituicytoma: overview of treatment strategies and outcome. Pituitary. 2012;15(2):227-36.

9. LISS L, KAHN EA. Pituicytoma, tumor of the sella turcica; a clinicopathological study. J Neurosurg. 1958;15(5):481-8.

10. Furtado SV, Ghosal N, Venkatesh PK, Gupta K, Hegde AS. Diagnostic and clinical implications of pituicytoma. J Clin Neurosci. 2010;17(7):938-43.

11. Katsuta T, Inoue T, Nakagaki H, Takeshita M, Morimoto K, Iwaki T. Distinctions between pituicytoma and ordinary pilocytic astrocytoma. Case report. J Neurosurg. 2003;98(2):404-6.

12. Benveniste RJ, Purohit D, Byun H. Pituicytoma presenting with spontaneous hemorrhage. Pituitary. 2006;9(1):53-8.

13. Hammoud DA, Munter FM, Brat DJ, Pomper MG. Magnetic resonance imaging features of pituicytomas: analysis of 10 cases. J Comput Assist Tomogr 2010;34:757-761.

14. Orrego JJ. Pituicytoma and isolated ACTH deficiency. Pituitary 2009;12:371372. 